\title{
Confiabilidad Operativa de Sistemas para Compresión de Gas y Generación Eléctrica en Complejos Petroleros
}

\author{
Marco P. Flores, José G. Torres, José H. Rodríguez y Agustín M. Alcaraz \\ Instituto de Investigaciones Eléctricas, Gerencia de Procesos Térmicos, \\ Reforma 113, Col. Palmira, C.P. 62490 Cuernavaca, Morelos-México \\ (e-mail: mpflores@iie.org.mx, jgtorres@iie.org.mx,jhrm@iie.org.mx,malcaraz@iie.org.mx)
}

Recibido Sept. 23, 2009; Aceptado Dic. 17, 2009; Versión Final recibida Feb. 24, 2010

\begin{abstract}
Resumen
Se presenta una comparación de tres alternativas tecnológicas para generación eléctrica y compresión de gas en complejos procesadores de aceite crudo, factibles de instalar en México. La comparación se realiza con base en los resultados de evaluaciones de confiabilidad operativa utilizando el método de diagramas de bloques. Se incluyen algunos conceptos relativos a la teoría de confiabilidad de sistemas y se define el tipo de modelo de confiabilidad utilizado, el cual corresponde a un arreglo en paralelo con redundancia en reserva pasiva y sin incluir mantenimiento en sus componentes. Asimismo, se describen las alternativas y los escenarios operativos analizados, se presentan los diagramas de bloques para cada alternativa, así como los resultados de confiabilidad, los cuales indican que el uso de motores eléctricos para compresión de gas y motores de combustión interna para generación eléctrica resulta más confiable que usar turbinas de gas.
\end{abstract}

Palabras clave: confiabilidad, diagramas de bloques, generación, compresión

\section{Operating Reliability of Gas Compression and Power Generation Systems in Offshore Oil Installations}

\begin{abstract}
This paper presents a study that compares operative results for three technological alternatives to produce electricity and compress gas in offshore crude oil processing facilities to be installed in Mexico. The comparison of alternatives is made based on system reliability estimations by using the "reliability block diagram" method. The fundamental concepts of the systems reliability theory are pointed out, and the reliability model is defined as a parallel arrangement with redundancy in passive reserve and without maintenance for any component. Likewise, the alternatives, and the analyzed operative scenarios are described, including the block diagrams and reliability results for each option. The results of the study show that using electrical motors to compress gas and internal combustion engines to produce electricity is more reliable than using gas turbines.
\end{abstract}

Keywords: reliability, block diagram, power generation, compression. 


\section{INTRODUCCIÓN}

La confiabilidad es un atributo fundamental para la operación segura y efectiva de cualquier sistema tecnológico moderno (Zio, 2009). En instalaciones costa afuera, las fallas de equipos críticos y los errores humanos han sido las causas principales de accidentes y paros forzados con impactos económicos, ambientales y afectaciones al personal operativo (Coco, 2003; Meshakti, 2007). De acuerdo a Meshakti (2007), una de las categorías principales de causas comunes de accidentes ocurridos en sistemas a gran escala es la carencia de análisis e involucramiento del personal y la organización en la etapa de diseño del sistema.

El diseño de sistemas mecánicos en plataformas petroleras debe considerar aspectos tales como la selección apropiada del equipo y su distribución, eficiencia, seguridad y compatibilidad con las características estructurales de la plataforma. Asimismo, para identificar y reducir la probabilidad de falla de equipos y sistemas, se deben llevar a cabo análisis de confiabilidad en forma conjunta con el proceso de diseño (Bergman, 1985). En la última década, en México se han estado analizando distintas opciones para la definición de procedimientos, modelos e infraestructura requerida para la separación, transporte a tierra y tratamiento de aceite crudo ligero y pesado, así como gas asociado, considerando la instalación de nuevos complejos de procesamiento en el área del Golfo de México (Huante, 2003; Rubio y Wen, 2004; Dantea et al., 2006; Nares et al., 2007). Respecto al procesamiento de gas, en los complejos de plataformas marinas típicamente se han utilizado sistemas de compresión mediante turbocompresores en los que se utiliza gas proveniente del paquete de endulzamiento localizado en la misma plataforma.

Actualmente, existe la alternativa de utilizar sistemas de compresión de gas con base en motores eléctricos provistos de variadores de velocidad y sistemas de generación de energía eléctrica. El propósito de este documento es comparar, desde el punto de vista operativo, tres alternativas tecnológicas de procesamiento, realizando evaluaciones de confiabilidad utilizando el método de diagramas de bloques (Haitao y Xianhui, 2007). El término confiabilidad se define en un sentido formal como la probabilidad de que los componentes o sistemas realicen satisfactoriamente sus funciones asignadas y dentro de los límites de ejecución especificados a una edad dada, para un determinado tiempo de misión, cuando se use en forma adecuada y para el propósito para el cual fueron destinados, manteniéndose los niveles de esfuerzo para las aplicaciones y ambientes de operación especificados (Kececioglu, 2003).

En este artículo se presentan los conceptos básicos para el cálculo de la confiabilidad de sistemas con diagramas de bloques, se establecen las bases para los análisis y se define el tipo de modelo de confiabilidad a utilizar. Asimismo, se describen las alternativas para compresión de gas y generación eléctrica, así como los escenarios operativos considerados en la evaluación; se definen los diagramas de bloques para las alternativas, así como las tasas de fallas para los diferentes equipos o componentes involucrados y, finalmente, se presentan los resultados de confiabilidad.

\section{DIAGRAMAS DE BLOQUES DE CONFIABILIDAD}

Los diagramas de bloques (DB) son una representación común para calcular la confiabilidad de sistemas (Xu et al., 2009). Esta representación se complementa con técnicas estadísticas para evaluar los parámetros de la mejor función densidad de probabilidad que represente el tiempo entre fallas. En forma resumida el método de DB consiste en obtener el valor de confiabilidad de cada elemento del sistema y posteriormente se encuentra la confiabilidad correspondiente a cada subsistema, al reducir el sistema a un arreglo equivalente conforme a los sistemas en serie y paralelo que lo constituyen.

\section{Sistemas en Serie y Paralelo}

La configuración de un sistema en serie es probablemente el modelo más común y el más sencillo de analizar. En un sistema en serie, todos los componentes (subsistemas) deben operar exitosamente para que el sistema funcione. En la Fig. 1 se presenta el diagrama de bloques de confiabilidad (DBC) para dicho sistema. 


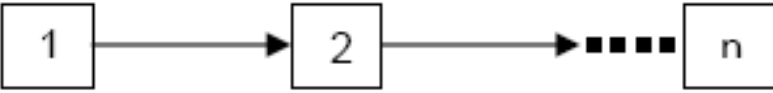

Fig. 1: DBC de un sistema en serie.

Si existe independencia estocástica en el funcionamiento de los componentes, entonces para un sistema en serie:

$R_{s}=P\left(E_{1}\right) P\left(E_{2}\right) \ldots P\left(E_{n}\right)$

$R_{s}=\prod_{i=1}^{n} R_{i}$

$Q_{s}=1-R s$

Donde:

$E_{i}=$ Evento "el $i$-ésimo subsistema opera exitosamente"

$R_{i}=P\left(E_{i}\right)=$ Confiabilidad del $i$-ésimo subsistema

$R_{S}=$ Confiabilidad del sistema

$Q_{s}=$ Probabilidad de falla del sistema

Puesto que $0 \leq R_{i} \leq 1$, para un sistema en serie la confiabilidad del sistema decae rápidamente, conforme se incrementa el número de componentes y será siempre menor o igual que la del componente menos confiable, esto es: $R_{s} \leq \operatorname{mí}\left\{R_{i}\right\}$ (Rausand y Høyland, 2004).

Un sistema en paralelo se considera en operación a menos que fallen todos sus componentes. En la Fig. 2 se muestra el diagrama de bloques para este tipo de sistemas.

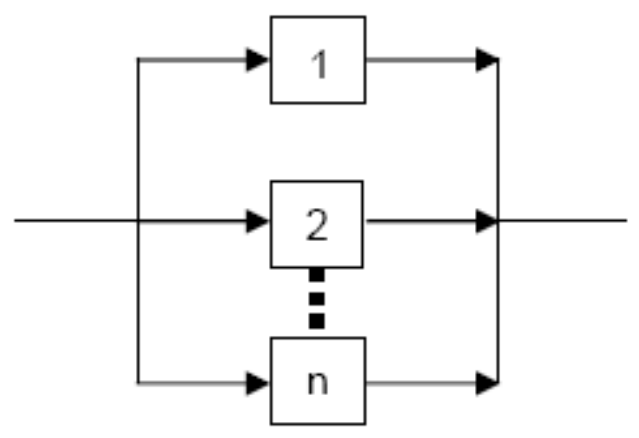

Fig. 2: DBC de un sistema en paralelo.

Nuevamente, suponiendo independencia y que los componentes no pueden ser reparados durante el período de misión:

$Q_{s}=\left[1-P\left(E_{1}\right)\right]\left[1-P\left(E_{2}\right)\right] \ldots\left[1-P\left(E_{n}\right)\right]$

$Q_{s}=\prod_{i=1}^{n}\left(1-R_{i}\right)$

$R_{s}=1-\prod_{i=1}^{n}\left(1-R_{i}\right)$

Por otra parte, las combinaciones serie-paralelo se analizan fácilmente al transformar algunos 
subsistemas en componentes equivalentes en serie o en paralelo (Levitin y Amari, 2009). Por ejemplo, para calcular la confiabilidad del sistema conformado por el arreglo en serie de los subsistemas paralelos mostrados en la Fig. 3, primero se transforman los subsistemas en paralelo a componentes equivalentes en serie.

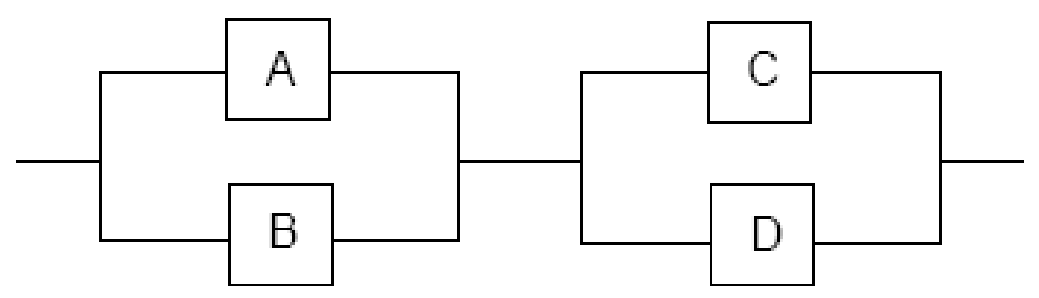

Fig. 3: Sistema serie-paralelo.

Suponiendo que $R_{A}=0.9, R_{B}=0.8, R_{C}=0.7, R_{D}=0.6$. Entonces, las confiabilidades de los componentes equivalentes son: $R_{A B}=0.98$ y $R_{C D}=0.88$. Con lo cual la confiabilidad del sistema será: $R_{S}=0.8624$.

Un segundo arreglo se muestra en la Fig. 4, donde dos subsistemas en serie se encuentran conectados en paralelo. El procedimiento en este caso consiste en transformar los subsistemas en serie en componentes en paralelo; obteniéndose las confiabilidades siguientes: $R_{A C}=0.63$ y $R_{B D}=$ 0.48. Con lo cual la confiabilidad del sistema será: $R_{S}=0.8076$.

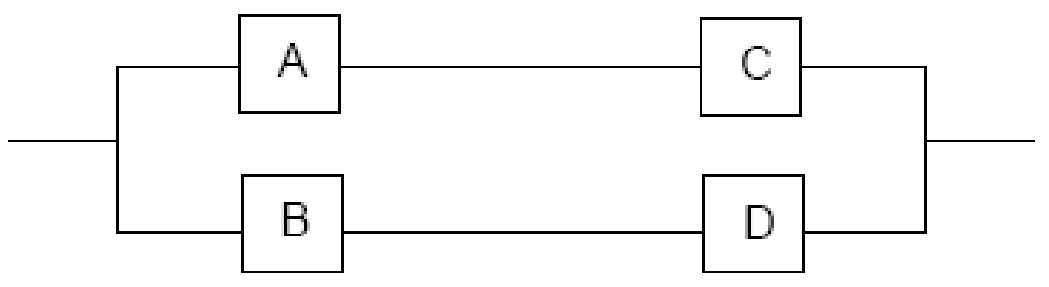

Fig. 4: Sistema paralelo-serie.

\section{Sistemas en Paralelo con Redundancia}

Un arreglo con redundancias se tiene cuando un componente o unidad esta en operación y uno o más de sus elementos están en reserva para entrar en operación cuando el primero falla. En estos casos se considera que los componentes, en operación y reserva, tienen las mismas tasas de falla, ya que normalmente se utilizan instrumentos de detección de falla e interruptores de arranque/paro que usualmente se consideran como $100 \%$ confiables.

Los sistemas en paralelo sujetos a mantenimiento se pueden clasificar de la siguiente forma (Sánchez et al; 1996):

a) Con mantenimiento de componentes mientras el sistema opera.

b) Con mantenimiento de componentes cuando el sistema está fuera de servicio.

El primer tipo de sistemas en paralelo es el que más se aproxima a la operación de las instalaciones reales; razón por la cual, será el usado en el modelo para estimar la confiabilidad de los sistemas de compresión y generación. Asimismo, para llevar a cabo el cálculo de la confiabilidad de estos sistemas, es necesario diferenciar los casos en los cuales los elementos redundantes se encuentran en reserva activa o pasiva. 


\section{BASES DE ANÁLISIS Y DEFINICIÓN DEL MODELO}

En el análisis desarrollado se considera que los equipos se encuentran en el estado de vida útil, por lo cual el modelo probabilista se ajusta a una distribución exponencial y queda determinado con un solo parámetro que no es función del tiempo, conocido como tasa de fallas ( $\lambda$ ); por lo que la confiabilidad de un componente se puede expresar como (Levitin y Amari, 2009; Smith, 1999):

$R_{i}(t)=e^{-\lambda t}$

Donde:

$R_{i}(t)=$ Confiabilidad del componente en función del tiempo.

$\mathrm{t} \quad=$ Tiempo de misión.

$\lambda=F / T_{r}=$ Tasa de fallas.

e $\quad=$ Base de los logaritmos neperianos $(2.718281 \ldots)$.

$\mathrm{F} \quad=$ Número de fallas en el periodo de tiempo considerado.

$\mathrm{T}_{\mathrm{r}} \quad=$ Tiempo real de operación en el periodo de tiempo considerado.

Por su parte, la confiabilidad de sistemas en paralelo con $\mathrm{n}$ componentes y redundancia activa sin mantenimiento está dada por la ecuación (6) (Smith, 1999); mientras que para sistemas con redundancia en espera se usa la siguiente expresión (Smith, 1999):

$R_{s}=R(t)=e^{-\lambda t}\left(1+\lambda t+\frac{\lambda^{2} t^{2}}{2 !} \ldots \frac{\lambda^{(n-1)} t^{(n-1)}}{(n-1) !}\right)$

Para definir el modelo que se utilizará para estimar la confiabilidad de las alternativas para compresión de gas y generación eléctrica, primeramente se analizaron los resultados de confiabilidad de sistemas sencillos en los cuales se considera que existe redundancia activa 0 pasiva, sin y con mantenimiento. Los casos analizados son arreglos de tres componentes instalados en paralelo en los cuales se requiere que operen dos elementos para que el sistema funcione. Los modelos están basados en distribuciones exponenciales para el tiempo entre fallas y para el tiempo de reparación, considerando tasas de falla de $\lambda=0.01$ y tasas de mantenimiento de $\mu=0.20$.

\section{Descripción de los Modelos Analizados y Función de Confiabilidad}

Modelo 1: Tres componentes, con uno en redundancia activa, sin mantenimiento.

$R_{s}=3 e^{-\lambda t}-3 e^{-2 \lambda t}+e^{-3 \lambda t}$

Modelo 2: Tres componentes, con uno en redundancia en espera, sin mantenimiento.

$R s=e^{-\lambda t}+\lambda t e^{-\lambda t}+\frac{1}{2} \lambda^{2} t^{2} e^{-\lambda t}$

Modelo 3: Tres componentes, con uno en redundancia activa, con mantenimiento únicamente a una unidad.

$R_{s}=\frac{-s_{2} s_{3}\left(s_{2}-s_{3}\right) e^{-\lambda_{1} t}+s_{1} s_{3}\left(s_{1}-s_{3}\right) e^{-\lambda_{2} t}-s_{1} s_{2}\left(s_{1}-s_{2}\right) e^{-\lambda_{3} t}}{\left(s_{1}-s_{2}\right)\left(s_{3}-s_{2}\right)\left(s_{1}-s_{3}\right)}$

Donde $s_{1}, s_{2}$ y $s_{3}$ corresponden a tres raíces de la ecuación:

$s^{3}+s^{2}(6 \lambda+2 \mu)+s\left(11 \lambda^{2}+4 \mu \lambda+\mu^{2}\right)+6 \lambda^{3}$ 
Modelo 4: Tres componentes, con uno en redundancia en espera, con mantenimiento únicamente a una unidad.

$R_{s}=\frac{-s_{2} s_{3}\left(s_{2}-s_{3}\right) e^{-\lambda_{1} t}+s_{1} s_{3}\left(s_{1}-s_{3}\right) e^{-\lambda_{2} t}-s_{1} s_{2}\left(s_{1}-s_{2}\right) e^{-\lambda_{3} t}}{\left(s_{1}-s_{2}\right)\left(s_{3}-s_{2}\right)\left(s_{1}-s_{3}\right)}$

Donde $s_{1}, s_{2}$ y $s_{3}$ corresponden a tres raíces de la ecuación:

$s^{3}+s^{2}(3 \lambda+2 \mu)+s\left(3 \lambda^{2}+2 \mu \lambda+\mu^{2}\right)+\lambda^{3}$

Resultados de confiabilidad de los modelos

En la tabla 1 se muestran los resultados obtenidos para la confiabilidad de los cuatro modelos descritos.

Tabla 1: Comparación de varios modelos de arreglos en paralelo con tres componentes.

\begin{tabular}{|c|c|}
\hline MODELO & $\begin{array}{c}\text { CONFIABILIDAD } \\
(\text { para } t=100)\end{array}$ \\
\hline 1) Redundancia activa sin mantenimiento & 0.750 \\
\hline 2) Redundancia en espera sin mantenimiento & 0.920 \\
\hline 3) Redundancia activa con mantenimiento a una unidad & 0.990 \\
\hline 4) Redundancia en espera con mantenimiento a una unidad & 0.998 \\
\hline
\end{tabular}

Como se observa en la tabla 1, la confiabilidad del sistema aumenta cuando se tiene redundancia y se cuenta con mantenimiento para uno de sus componentes. Si se tuviera la posibilidad de dar mantenimiento múltiple, es decir a dos o más componentes de manera simultánea, la confiabilidad se incrementaría aún más.

Considerando que en las plataformas marinas existen limitaciones de recursos de mantenimiento mayor de los equipos, como una medida conservadora para evaluar la confiabilidad global del complejo petrolero para fines de comparación de las alternativas de compresión de gas y generación eléctrica, se establece que los sistemas con redundancia se resuelven considerando que los componentes redundantes se encuentran en reserva pasiva (en espera) y sin incluir mantenimiento de sus componentes.

Por lo tanto, los tipos de redundancia que se utilizarán en las alternativas analizadas para las plataformas de producción y compresión, son: 1) para los sistemas de endulzamiento, acondicionamiento, generación eléctrica en plataforma de producción y enlace eléctrico entre plataformas de compresión y producción, se tiene un arreglo de dos componentes en paralelo de los cuales uno está en reserva, 2) para los módulos de turbo-compresión y moto-compresión se tiene un arreglo de cuatro componentes en paralelo incluyendo uno en reserva, 3) para el sistema de motobombas de transporte de aceite se tiene un arreglo de cinco bombas con una de ellas en reserva, 4) para los moto-generadores se tienen arreglos de seis equipos con tres de ellos en reserva para la plataforma de producción y nueve equipos con tres en reserva para la plataforma de compresión.

\section{ALTERNATIVAS Y ESCENARIOS EVALUADOS}

La definición de alternativas para generación eléctrica y compresión de gas se llevó a cabo partiendo del siguiente esquema general de procesamiento de las corrientes de crudo (Estrada et al., 2000; Kettles, 2001; PEMEX, 2008). El complejo estaría compuesto por cuatro plataformas: una plataforma de Producción, una de Compresión, una de Enlace y una Habitacional. En la plataforma de 
Producción se realizaría la separación de los flujos de gas y aceite y el bombeo de aceite a través de un arreglo de cuatro módulos de bombeo más uno de respaldo. En la plataforma de Compresión se realizaría la compresión de gas y recuperación de condensados a través de tres módulos de compresión más uno de respaldo.

\section{Descripción de alternativas}

Se definieron tres alternativas de esquemas operativos, para las cuales se evaluaron los índices de confiabilidad mediante el método de diagramas de bloques; las diferencias entre las alternativas son básicamente en el tipo de tecnología a utilizar tanto en los módulos de compresión (turbo-compresión o moto-compresión) como en la generación de energía eléctrica (turbo-generación o motogeneración).

En la tabla 2 se describen las características de los equipos para compresión de gas, transferencia de aceite y generación eléctrica, así como las condiciones operativas de los equipos para cada alternativa.

Tabla 2: Alternativas para generación eléctrica y compresión de gas.

\begin{tabular}{|c|c|c|}
\hline ALTERNATIVA & DESCRIPCIÓN & CONDICIONES NOMINALES \\
\hline $\begin{array}{l}\text { Alternativa } 1 . \\
\text { Convencional: } \\
\text { Con turbo-compresión y } \\
\text { turbo-generación }\end{array}$ & $\begin{array}{l}\text { Turbocompresores a gas para } \\
\text { módulos de la plataforma de } \\
\text { Compresión, motobombas con } \\
\text { variadores de velocidad para la } \\
\text { transferencia del aceite en } \\
\text { plataforma de Producción y } \\
\text { turbogeneradores para energía } \\
\text { eléctrica en la plataforma de } \\
\text { Producción. }\end{array}$ & $\begin{array}{l}\text { a) Dos endulzadoras de gas, una en } \\
\text { operación. } \\
\text { b) Un secador mecánico de gas y un } \\
\text { acondicionador eléctrico de gas, uno } \\
\text { de ellos en operación. } \\
\text { c) Dos turbogeneradores, uno en } \\
\text { operación. } \\
\text { d) Cinco motobombas, cuatro en } \\
\text { operación. } \\
\text { e) Cuatro módulos de compresión, tres } \\
\text { en operación. }\end{array}$ \\
\hline $\begin{array}{l}\text { Alternativa } 2 \text {. } \\
\text { Con moto-compresión y } \\
\text { turbo-generación }\end{array}$ & $\begin{array}{l}\text { Motocompresores } \text { eléctricos } \\
\text { con variadores de velocidad } \\
\text { para módulos de la Plataforma } \\
\text { de Compresión, motobombas } \\
\text { con variadores de velocidad } \\
\text { para la transferencia del aceite } \\
\text { en Plataforma de Producción y } \\
\text { turbogeneradores de energía } \\
\text { eléctrica en ambas plataformas } \\
\text { (Producción y Compresión). }\end{array}$ & $\begin{array}{l}\text { a) Dos endulzadoras de gas, una en } \\
\text { operación. } \\
\text { b) Un secador mecánico de gas y un } \\
\text { acondicionador eléctrico de gas, uno } \\
\text { de ellos en operación. } \\
\text { c) Dos turbogeneradores en plataforma } \\
\text { de Producción, uno en operación. } \\
\text { d) Cinco motobombas, cuatro en } \\
\text { operación. } \\
\text { e) Tres turbogeneradores en plataforma } \\
\text { de Compresión, dos en operación. } \\
\text { f) Cuatro módulos de compresión, tres } \\
\text { en operación. }\end{array}$ \\
\hline $\begin{array}{l}\text { Alternativa } 3 \text {. } \\
\text { Con moto-compresión y } \\
\text { moto-generación }\end{array}$ & $\begin{array}{l}\text { Motocompresores con } \\
\text { variadores de velocidad para } \\
\text { módulos de la Plataforma de } \\
\text { Compresión, motobombas con } \\
\text { variadores de velocidad para la } \\
\text { transferencia del aceite en } \\
\text { Plataforma de Producción y } \\
\text { generadores con base en } \\
\text { motores de combustión para } \\
\text { generación de energía eléctrica } \\
\text { en ambas plataformas } \\
\text { (Producción y Compresión). }\end{array}$ & $\begin{array}{l}\text { a) Un sistema de acondicionamiento de } \\
\text { gas amargo para motogeneradores. } \\
\text { b) Seis motogeneradores en plataforma } \\
\text { de Producción, tres en operación. } \\
\text { c) Cinco motobombas, cuatro en } \\
\text { operación. } \\
\text { d) Nueve motogeneradores de en } \\
\text { plataforma de Compresión, seis en } \\
\text { operación. } \\
\text { e) Cuatro módulos de compresión, tres } \\
\text { en operación. }\end{array}$ \\
\hline
\end{tabular}




\section{Diagramas de bloques}

Para cada una de las alternativas se elaboró el diagrama de bloques de confiabilidad del sistema correspondiente. En las Fig. 56 y 7 se muestran estos diagramas, mientras que en la tabla 3 se describe la nomenclatura empleada.

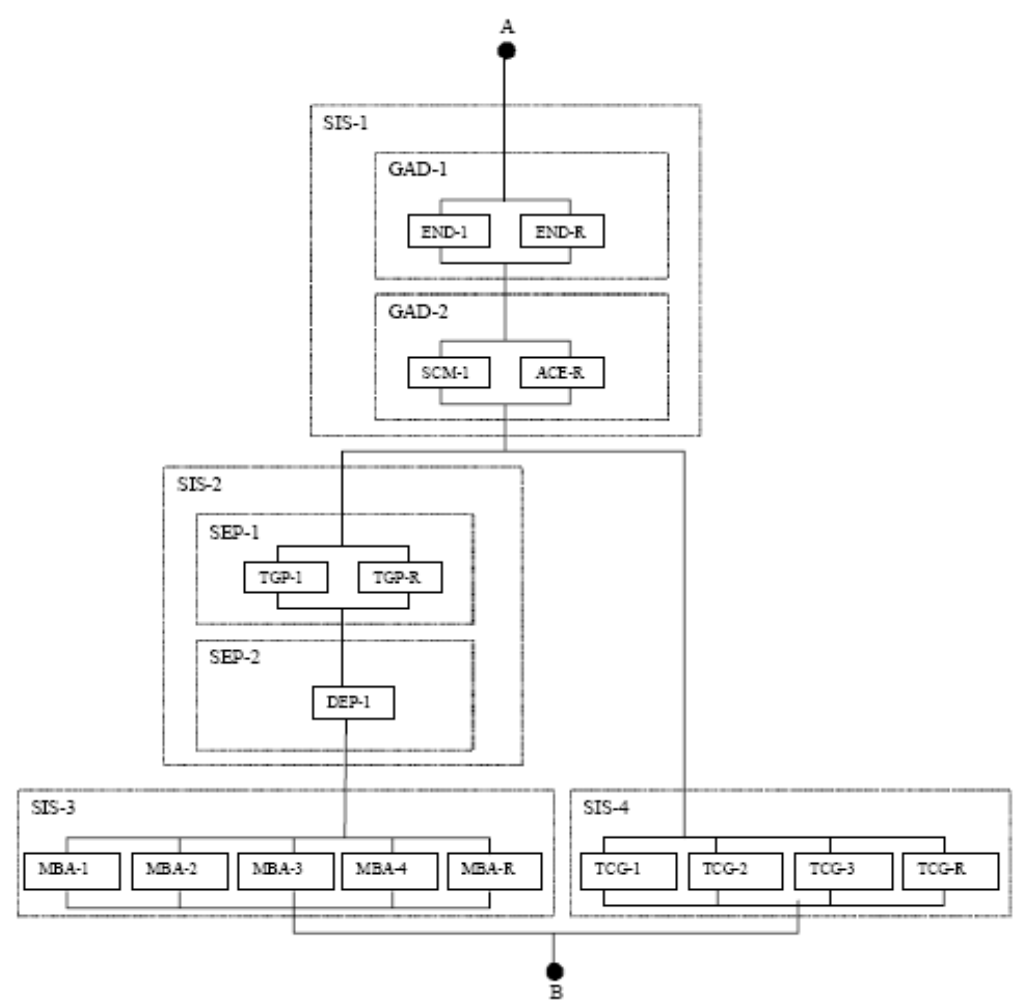

Fig. 5: Diagrama de bloques de alternativa 1 (turbo-compresión y turbo-generación).

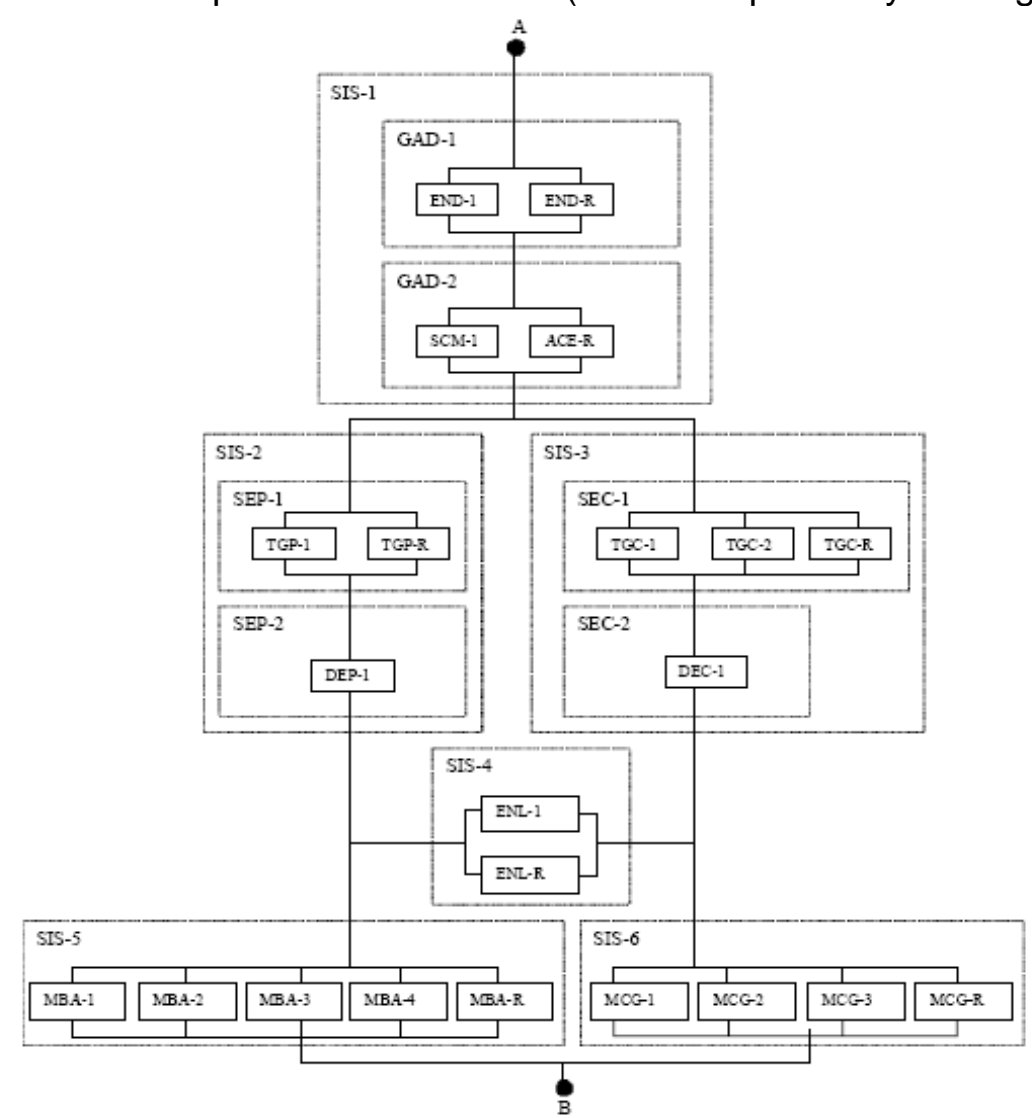

Fig. 6: Diagrama de bloques de alternativa 2 (moto-compresión y turbo-generación). 


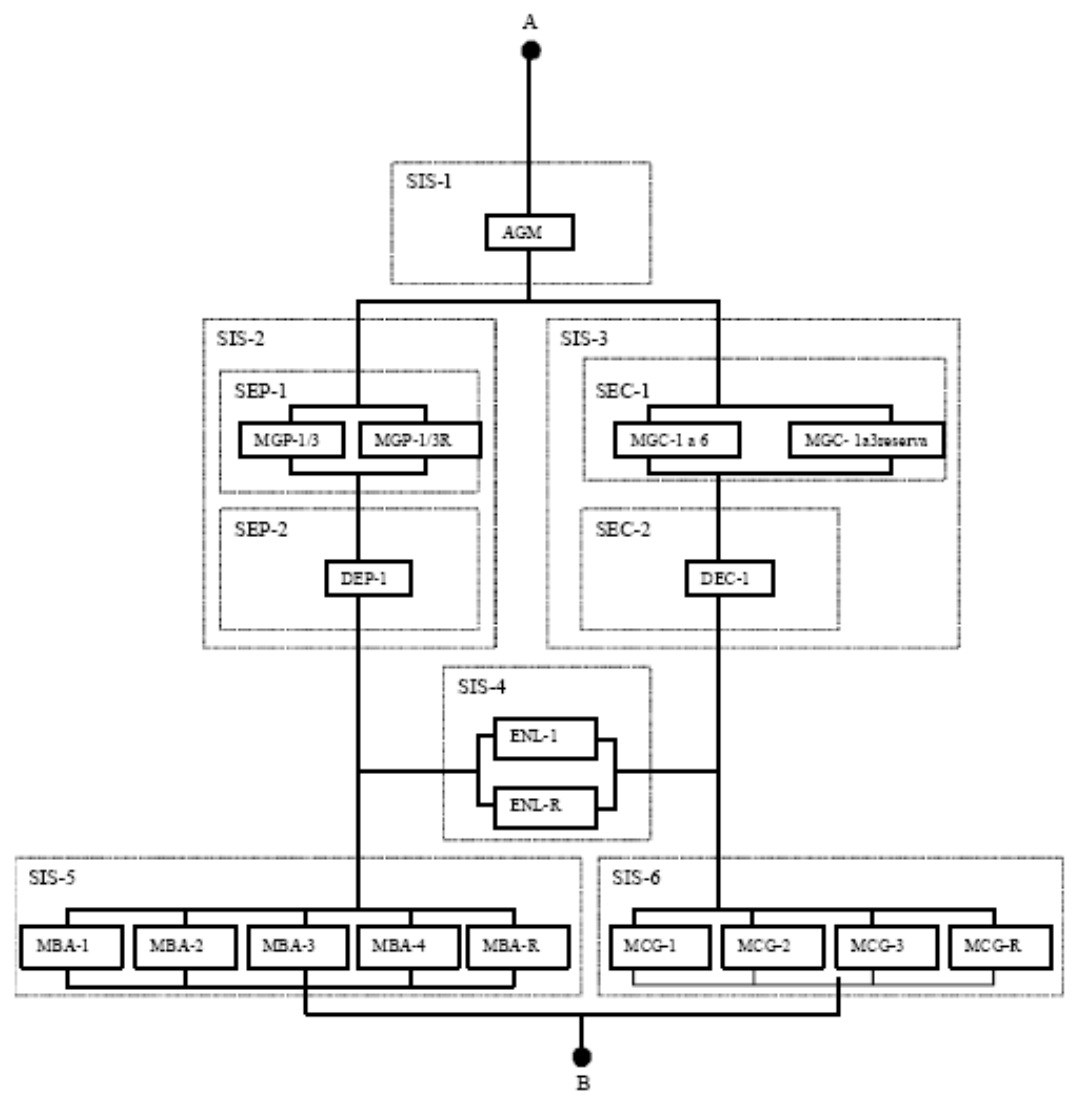

Fig. 7: Diagrama de bloques de alternativa 3 (moto-compresión y moto-generación).

Tabla 3: Nomenclatura utilizada en los diagramas de bloques de confiabilidad.

\begin{tabular}{|c|c|c|c|}
\hline \multicolumn{2}{|r|}{ Bloques básicos } & \multicolumn{2}{|r|}{ Bloques de agrupación } \\
\hline CLAVE & DESCRIPCIÓN & CLAVE & DESCRIPCIÓN \\
\hline END & Endulzadora de gas & GAD & Producción de gas dulce combustible \\
\hline SCM & Secado mecánico de gas & SEP & $\begin{array}{l}\text { Generación y distribución de energía } \\
\text { eléctrica en P. Producción }\end{array}$ \\
\hline ACE & Acondicionamiento eléctrico de gas & SEC & $\begin{array}{l}\text { Generación y distribución de energía } \\
\text { eléctrica en P. Compresión }\end{array}$ \\
\hline AGM & $\begin{array}{l}\text { Acondicionamiento de gas para } \\
\text { motogeneradores }\end{array}$ & - \# & Número consecutivo de bloque \\
\hline TGP & $\begin{array}{l}\text { Turbogenerador a gas en P. } \\
\text { Producción }\end{array}$ & $-\mathrm{R}$ & Elemento de respaldo \\
\hline MGP & $\begin{array}{l}\text { Motogenerador a gas en P. } \\
\text { Producción }\end{array}$ & & \\
\hline TGC & $\begin{array}{l}\text { Turbogenerador a gas en } \mathrm{P} \text {. } \\
\text { Compresión }\end{array}$ & & \\
\hline MGC & $\begin{array}{l}\text { Motogenerador a gas en P. } \\
\text { Compresión }\end{array}$ & & \\
\hline DEP & $\begin{array}{l}\text { Distribución energía eléctrica en P. } \\
\text { Producción }\end{array}$ & & \\
\hline DEC & $\begin{array}{l}\text { Distribución energía eléctrica en P. } \\
\text { Compresión }\end{array}$ & & \\
\hline ENL & Enlace eléctrico entre plataformas & & \\
\hline MBA & Motobomba de transferencia de aceite & & \\
\hline MCG & Módulo de moto-compresión de gas & & \\
\hline TCG & Módulo de turbo-compresión de gas & & \\
\hline
\end{tabular}




\section{Escenarios}

La evaluación de las alternativas operativas se llevó a cabo para los dos escenarios siguientes:

1. Operación del complejo en condición de producción nominal. Operación de cuatro bombas de transferencia de aceite, tres módulos de compresión y los sistemas necesarios de apoyo (energía eléctrica, gas combustible, distribución de energía eléctrica, etc.); todos con disponibilidad de emplear sus equipos de relevo en condiciones de diseño.

2. Operación del complejo en condición de producción mínima (33 \% de capacidad). Operación de dos bombas de transferencia de aceite, un módulo de compresión y los sistemas necesarios de apoyo. En este escenario se consideró que el resto de los equipos que no están en operación se encuentran en estado de disponibilidad, o sea, que en caso de falla de uno de los equipos que operan se tiene disponible cualquiera de los que no están en operación.

\section{TASAS DE FALLA DE LOS COMPONENTES}

Para definir los valores de tasas de fallas para los diferentes equipos analizados, se utilizó la información de la Base de Datos de Confiabilidad para Instalaciones Costa Afuera (OREDA, 2002). Para los módulos de compresión con turbina de gas, se utilizó una tasa promedio de 10.88 fallas críticas por año, la cual es similar a la estimada con información estadística de módulos de compresión con turbina de gas en complejos petroleros mexicanos.

Respecto a la turbo-generación de energía eléctrica con ciclo abierto, la base de datos de OREDA (OREDA, 2002), indica una tasa de 4.81 fallas por año para instalaciones costa afuera, mientras que la experiencia en instalaciones en México indica una tasa de 4.2 fallas por año. Para fines de este estudio se utilizaron las tasas de falla de equipo similar al requerido en el futuro complejo petrolero, obtenidas del documento de OREDA (OREDA, 2002) exceptuando las tasas del enlace eléctrico y distribución de energía eléctrica, cuyos valores fueron propuestos independientemente. Los valores de las tasas de falla se presentan en la tabla 4.

Tabla 4: Tasas de falla utilizadas en el análisis de confiabilidad.

\begin{tabular}{|l|c|}
\hline \multicolumn{1}{|c|}{ EQUIPO O SUBSISTEMA } & TASA DE FALLAS \\
\hline Endulzadora de gas & 1.44 \\
\hline Secado mecánico de gas & 3.25 \\
\hline Acondicionamiento eléctrico de gas & 0.33 \\
\hline Turbogenerador a gas en producción o compresión & 4.81 \\
\hline Distribución energía eléctrica en producción o compresión & 0.1 \\
\hline Motobomba de transferencia de aceite & 2.14 \\
\hline Módulo de turbo-compresión de gas & 10.88 \\
\hline Enlace eléctrico entre plataformas & 0.1 \\
\hline Módulo de moto-compresión de gas & 2.17 \\
\hline Acondicionamiento para motogeneradores & 0.11 \\
\hline Motogenerador a gas en producción o compresión & 3.27 \\
\hline
\end{tabular}

\section{RESULTADOS}

Los resultados de confiabilidad de los dos escenarios analizados para diferentes periodos de misión, se obtuvieron mediante una hoja de cálculo en Excel desarrollada por los autores. En las tablas 5 y 6 y Figs. 8 y 9 , se muestran dichos resultados, en donde se observan mayores valores de confiabilidad para las alternativas que incluyen moto-compresión (alternativas 2 y 3) y una disminución drástica de la confiabilidad para la alternativa 1 a partir del primer mes de operación. 
Tabla 5: Confiabilidad del escenario en producción nominal.

\begin{tabular}{|c|c|c|c|}
\hline DÍAS & ALTERNATIVA 1 & ALTERNATIVA 2 & ALTERNATIVA 3 \\
\hline 1 & 100.00 & 100.00 & 100.00 \\
\hline 5 & 99.91 & 99.97 & 100.00 \\
\hline 15 & 97.15 & 99.55 & 99.85 \\
\hline 30 & 83.41 & 96.47 & 98.26 \\
\hline 60 & 47.47 & 77.68 & 84.60 \\
\hline
\end{tabular}

Tabla 6: Confiabilidad del escenario en producción mínima (33\% de capacidad).

\begin{tabular}{|c|c|c|c|}
\hline DÍAS & ALTERNATIVA 1 & ALTERNATIVA 2 & ALTERNATIVA 3 \\
\hline 1 & 99.999 & 99.999 & 100.000 \\
\hline 5 & 99.980 & 99.980 & 100.000 \\
\hline 15 & 99.826 & 99.818 & 99.997 \\
\hline 30 & 99.250 & 99.233 & 99.982 \\
\hline 60 & 95.410 & 96.346 & 99.815 \\
\hline
\end{tabular}

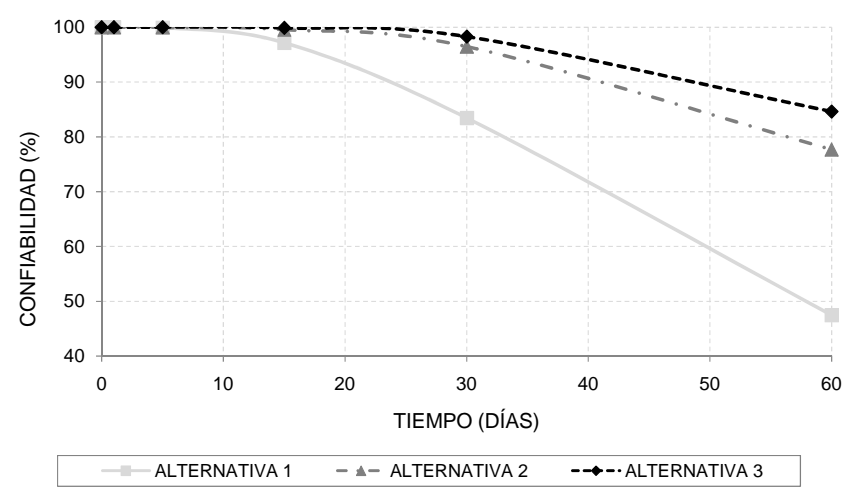

Fig. 8: Comparación de confiabilidad a condiciones de diseño (sin mantenimiento).

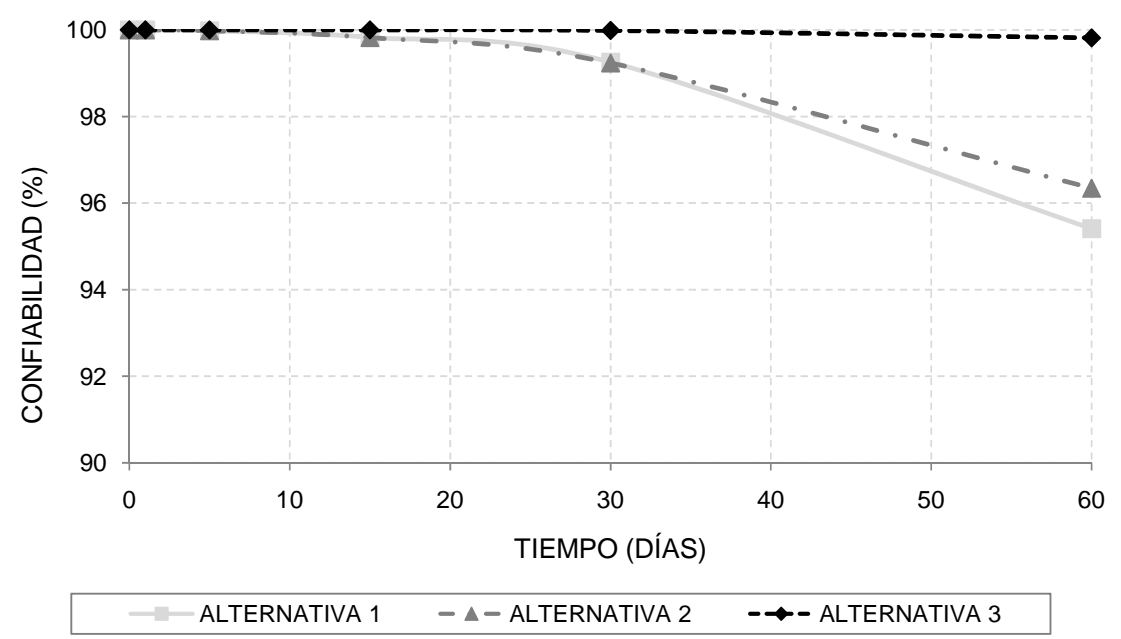

Fig. 9: Comparación de confiabilidad a $33 \%$ de capacidad (sin mantenimiento). 


\section{CONCLUSIONES}

Con base en los datos de tasa de falla utilizados, se concluye que el sistema de moto-compresión resulta más confiable que el uso de turbo-compresión.

Por otra parte, al comparar los resultados de las alternativas que utilizan turbo-generación eléctrica contra la que incluye moto-generación eléctrica, se concluye que el uso de motores de combustión interna para generación eléctrica conduce a sistemas más confiables, dada la capacidad unitaria y la redundancia para estos sistemas. Para el caso analizado, se requieren tres motores de combustión interna por cada módulo de turbo-generación eléctrica.

Finalmente, se concluye que la alternativa más confiable incluye el uso de generadores eléctricos con base en motores de combustión interna en las plataformas de Producción y Compresión, así como compresores de gas con base en motores eléctricos que incluyan variadores de velocidad en la Plataforma de Compresión.

Se debe considerar que, aún cuando el sistema a diseñar, por su ubicación, debe tener una configuración con una confiabilidad que garantice periodos de operación largos sin fallas de equipos y salidas forzadas; para la selección y diseño final de los sistemas a utilizar se deben considerar otros criterios tales como la correcta distribución de equipo, la eficiencia del sistema, los sistemas de seguridad y la compatibilidad con las características estructurales de la plataforma, entre otros.

\section{REFERENCIAS}

Bergman B., On Reliability Theory and its Applications, Scandinavian Journal of Statistics, 12(1), 1-41 (1985).

Coco J. C., Large Property Damage Losses in the Hydrocarbon-Chemical Industries, 20th Edition, 5156, Marsh's Risk Consulting Practice, New York (2003).

Dantea R. C., Geffroy-Aguilarb E. y Chávez A.E., Viscoelastic Models for Mexican Heavy Crude Oil and Comparison with a Mixture of Heptadecane and Eicosane. Part I, Fuel: 85(4), 559-568 (2006).

Estrada, A., Silva, L. M. y Contreras, R., Modernización y Optimización de los Esquemas de Proceso en Complejos Marinos de Producción - Alternativas en Beneficio del Ambiente, XXVII Congresso Interamericano de Engenharia Sanitária e Ambiental, 1-6, Porto Alegre, Brasil, 3 a 8 de diciembre (2000)

Haitao G. y Xianhui Y., A Simple Reliability Block Diagram Method for Safety Integrity Verification, Reliability Engineering and System Safety: 92(9), 1267-1273 (2007).

Huante, L., Asesoría Técnica en el Área de Ingeniería Mecánica para el Proyecto Crudo Ligero Marino, Informe No. IIE/44/12419/I 008/F, Instituto de Investigaciones Eléctricas Cuernavaca, Morelos, México (2003).

Kececioglu, D. B., Maintainability, Availability, and Operational Readiness Engineering Handbook, Vol. 1, pp. 26, DEStech Publications, Inc., Lancaster, Pennsylvania, USA (2003).

Kettles R., Kuo J. C. y Rubio J., Cantarell's Akal C Complex: the World's Largest Offshore Gas Treating and Lift Gas Generation Platforms, 2001 Offshore Technology Conference, Houston, Texas, USA, 30 de Abril a 3 de Mayo (2001).

Levitin G. y Amari S.V., Optimal Load Distribution in Series-Parallel Systems, Reliability Engineering and System Safety: 94(2), 254-260 (2009).

Meshakti, N. The Safety and Reliability of Complex Energy Processing Systems, Energy Sources, Part B: Economics, Planning, and Policy, 2(2), 141-154 (2007). 
Nares H.R., Schacht-Hernández P., Ramírez-Garnica M.A., Cabrera-Reyes M.C. y Noé-Valencia L., Heavy-Crude-Oil Upgrading With Transition Metals, 2007 SPE Latin American and Caribbean Petroleum Engineering Conference, Buenos Aires, Argentina, 15 a 18 de Abril (2007).

OREDA: OREDA-Offshore Reliability Data Handbook, 4th Edition, DNV - Det Norske Veritas, Oslo, Norway (2002).

PEMEX, Centro de proceso Akal C, en línea, México, Editor: Iván Á. Esquivel, última modificación: http://www.pemex.com/index.cfm?action=content\&sectionID=73\&catID=11958\&contentID=18248, junio de 2008, acceso: 2 de abril (2009). 77

Rausand, M. y Høyland A., System Reliability Theory: Models, Statistical Methods, and Applications, 2nd Edition, pp. 149, Wiley-Interscience, New York, USA (2003).

Rubio, J. J. y Wen Yu, Modelling of crude oil blending via discrete-time neural networks, International Conference on Electrical and Electronics Engineering (ICEEE) and X Conference on Electrical Engineering (CIE 2004), 427-432, Acapulco, Guerrero; Mexico, 8 a 10 de Septiembre (2004).

Xu H., L. Xing, y R. Robidoux, DRBD: Dynamic Reliability Block Diagrams for System Reliability Modelling, International Journal of Computers and Applications: 31(2), 132-141 (2009).

Sánchez, R., Torres, J.G. y Franco, J. M., Confiabilidad de componentes, sistemas y unidades de generación hidroeléctrica, Boletín IIE: 20(6), noviembre-diciembre (1996).

Smith, D. J., Reliability, Maintainability and Risk - Practical Methods for Engineers, 7th Edition, 10-94, Elsevier Butterworth-Heinemann, Burlington, MA, USA (2005).

Zio, E., Reliability engineering: Old problems and new challenges, Reliability Engineering and System Safety: 94(2), 125-141 (2009). 